\title{
BMJ Open Rural longitudinal integrated clerkships and medical workforce outcomes: a scoping review protocol
}

\author{
Jessica Beattie (D) , ${ }^{1}$ Marley J Binder, ${ }^{2}$ Lara Fuller ${ }^{3}$
}

To cite: Beattie J, Binder MJ, Fuller L. Rural longitudinal integrated clerkships and medical workforce outcomes: a scoping review protocol. BMJ Open 2022;12:e058717. doi:10.1136/ bmjopen-2021-058717

- Prepublication history and additional supplemental material for this paper are available online. To view these files, please visit the journal online (http://dx.doi.org/10.1136/ bmjopen-2021-058717).

Received 31 October 2021 Accepted 03 February 2022

Check for updates

(c) Author(s) (or their employer(s)) 2022. Re-use permitted under CC BY-NC. No commercial re-use. See rights and permissions. Published by BMJ.

${ }^{1}$ Rural Community Clinical School, Deakin University, Warrnambool, Victoria, Australia ${ }^{2}$ Deakin Rural Health, Deakin University, Geelong, Victoria, Australia

${ }^{3}$ Rural Community Clinical School, Deakin University, Colac, Victoria, Australia

Correspondence to

Jessica Beattie;

j.beattie@deakin.edu.au

\section{ABSTRACT}

Introduction The shortage of doctors in rural locations is an international problem, contributing to limited access to healthcare and a health disparity between rural and metropolitan populations. To encourage additional doctors to work in rural locations, more doctors than ever are being trained in rural settings. One rural clerkship model that is gaining recognition for fostering rural careers is the Longitudinal Integrated Clerkship. Longitudinal Integrated Clerkship programmes vary in terms of settings and durations, but at their core have the fundamental commonality of continuity, with students learning the curriculum in an integrated manner. The scoping review will synthesise the literature pertaining to medical workforce outcomes of rural Longitudinal Integrated Clerkship programmes, to uncover areas that require further research and establish elements of medical education programme design that positively influence rural workforce outcomes.

Methods and analysis The review will follow Arksey and 0'Malley's six step scoping review framework. MEDLINE, CINAHL complete (EBSCOhost), Scopus, Embase (Elsevier) and ISI Web of Science databases will be searched along with Google, Google Scholar, ProQuest and WHO library database. Single design studies examining the geographic work locations and/or medical specialty of rural Longitudinal Integrated Clerkship graduates will be included. Data from quantitative and mixed-methods studies will be included. Only studies written in English will be included. There will be no date range restriction imposed on the reviewed studies. Two reviewers will independently screen and critically appraise the articles to determine if they meet the inclusion criteria. Data from eligible studies will be extracted for synthesis.

Ethics and dissemination Scoping reviews do not require ethics approval. Results will be submitted to a peer-reviewed journal and may be presented at relevant conferences. The findings will also be shared within the Longitudinal Integrated Clerkship community of medical educators.

\section{INTRODUCTION}

The maldistribution of the medical workforce is a global issue, with a myriad of policies and programmes developed to redress this problem and encourage more doctors to work in rural locations. ${ }^{12}$ Within Canada, only $8 \%$ of doctors work in rural locations

\section{Strengths and limitations of this study}

To the best of our knowledge, this will be the first scoping review to focus on rural Longitudinal Integrated Clerkships and medical workforce outcomes, seeking to find evidence to mitigate the international problem of medical workforce maldistribution.

- The review will follow a rigorous methodology, with the search strategy developed in conjunction with an experienced librarian.

- Formal evaluation of the evidence will not be undertaken; however, the review will identify gaps in the literature and generate recommendations for future research.

- A limitation is only articles published in English will be considered in the review.

despite approximately $19 \%$ of the population residing rurally. ${ }^{3}$ While in Australia, $79 \%$ of doctors work in a metropolitan setting, equating to 454.5 doctors per 100000 compared with 407.3 in larger rural towns and 119.9 in small rural towns. ${ }^{4}$

The maldistribution of the medical workforce is not only geographic, but there is now a growing medical specialty divide, with the number of doctors training to work in primary care decreasing and interest in subspecialisation increasing. ${ }^{56}$ Since 2013, the number of Australian subspecialists has grown at three times the rate of generalists. ${ }^{4}$ Rural communities require general practice (GP) and non-GP specialists with a broad range of generalist skills to meet their community's healthcare needs. ${ }^{4}$ Uneven geographical and specialist distribution limits access to healthcare services in rural communities, exacerbating the health status disparity between rural and urban communities, which includes higher mortality rates and lifestyle risk factors, such as smoking, excessive alcohol consumption and levels of obesity. ${ }^{7}$ The potentially avoidable death rate in Australian rural communities is between $20 \%$ and $65 \%$ higher than metropolitan communities. ${ }^{4}$ 
One of the fundamental polices many countries use to strengthen the rural medical workforce is to embed medical students in rural longitudinal training, with countries such as Australia mandating that $25 \%$ of commonwealth supported medical students must be trained in a rural location for at least 12 months. ${ }^{8}$ Rural medical training is not homogeneous, with two clerkship models prevalent, traditional block rotations (BRs) and longitudinal integrated clerkships (LICs). These clerkship models are inherently different despite often delivering the same curriculum and assessments within the same medical degree. ${ }^{9}$ LIC programmes can occur across a variety of settings and locations but are more often based in small rural communities attached to a GP/primary care setting and smaller rural health service. ${ }^{10}$ LIC students learn the curriculum in an integrated manner, gaining exposure to all the required disciplines simultaneously. ${ }^{9}{ }^{10}$ Rural BR clerkships are discipline-specific short-term rotations predominately based in hospitals. ${ }^{11}$

LIC programmes have gained popularity within medical education over the preceding decades and are estimated to have doubled globally between 2010 and $2015 .{ }^{10} \mathrm{~A}$ catalyst for the development and international uptake of LIC programmes was to address medical workforce shortages in rural settings. ${ }^{10}$ The LIC International body, The Consortium of Longitudinal Integrated Clerkships (CLIC) developed a consensus statement on elements that must be present to be considered a LIC programme. Three fundamental components were agreed on (i) medical students participate in the comprehensive care of patients' over time, (ii) medical students have continuing relationships with these patients clinicians and (iii) medical students meet most the year's core clinical competencies across multiple discplines simultaneously. ${ }^{12}$

The initial LIC-related literature focused on confirming the educational benefits and equivalence of the LIC clerkship model when compared with the BR clerkship. ${ }^{13} 14$ Overwhelmingly, LIC programmes have been found to achieve academic equivalence with BR's. ${ }^{1315}$ Subsequently, LIC-related research expanded focus, finding that LIC programmes foster the development of trusting, continuous relationships with supervisors, healthcare teams and patients, allow students greater access to patients, support active participation in patient care, enhance confidence in clinical skills and heightened preparedness for practice. ${ }^{16-20}$

Reviews of rural workforce outcomes from extended rural medical training have predominately analysed programme outcomes, without detailed reference to clerkship model. A 2018 scoping review found that when rural immersion programmes such as BRs and LICs are viewed through the same lens, they are moderately successful in increasing the rural supply of doctors, with authors stating that evidence related to the programme design that is most effective in achieving this outcome is still underdeveloped. ${ }^{21}$ As such, the differences in programme design between the two clerkship models require their relative influence on rural workforce outcomes to be examined.
Over recent years, many rural LIC programmes have reached a level of maturity where graduating cohorts are likely to have completed vocational medical training. Emerging evidence from single site studies, with a variety of methodological approaches have found that rural LICs are effective in influencing rural workforce outcomes. ${ }^{2-25}$ Studies have reported findings in a variety of ways, including geographic work locations (metropolitan/rural) compared with other training models; workforce locations of graduates who undertake GP; analysis of specific postgraduate years and analyses of the effect of graduates' other clinical training, in conjunction with the rural LIC year. ${ }^{23}{ }^{24}{ }^{26}$ Medical specialty choice of rural LIC graduates has been examined by a limited number of studies and has primarily focused on graduates who specialise in primary care, as this is the foundation of rural healthcare delivery. ${ }^{26} 27$ The varied comparative approaches and analyses require mapping.

Moreover, as rural LIC programmes can vary greatly in length, setting and student selection policies, a focused synthesis is required to determine specific patterns associated with rural workforce outcomes and importantly to determine any reported medical programme design elements within the clerkship model that may facilitate this. ${ }^{10}$ Therefore, this review will synthesise the evidence associated with the geographic and career workforce outcomes of graduates who have participated in a rural LIC programme and identify programme design elements that may facilitate positive rural workforce outcomes.

To undertake the review, a preliminary search of PROSPERO, MEDLINE, the Cochrane Database of Systematic Reviews and the JBI Database of Systematic Reviews and Implementation Reports was conducted, finding that there are currently no previous or under-development systematic reviews specifically on the topic identified. Specific LIC programme research has been synthesised by three narrative reviews (Walters et al (2012), Brown et al (2019) and Bartlett et al (2020)). A review by Thistlewaite et al (2013) although centred on longitudinal placements did not solely focus on rural LIC-specific literature and set the minimum placement duration as 13 weeks, which is in contrast with the CLIC typology that requires the student to meet the majority of the years learning within the programme. ${ }^{14}$ Brown et al focused on the development and implementation of LICs, while Barlett et al reviewed how to develop sustainable LIC clerkships. ${ }^{28}{ }^{29}$ The reviews of Walters $e t$ al and Thistlewaite et al briefly examined career and workforce outcomes of LIC programmes, but there were only a very small number of papers, published at least 10 years ago available for inclusion at the time. ${ }^{13}{ }^{14}$ Walters et al found that LIC students were positively influenced towards rural career choices but did not delve into the factors that influenced this, stating that they are "complex'. ${ }^{13}$ Thistlewaite $e t$ al found that rural LIC programmes improved attitudes towards rural practice but did not focus on actual rural workforce outcomes. $^{14}$ 
The scoping review will synthesise the international literature pertaining to medical workforce outcomes of rural LIC programmes, to uncover areas that require further research and establish elements of medical education programme design that positively influence rural workforce outcomes.

\section{METHODS AND ANALYSIS \\ Methodological framework}

The scoping review will be reported in accordance with the preferred reporting items for systematic reviews and metaanalysis extension for scoping reviews (PRISMA-ScR) ${ }^{30}$

The methodological framework employed will be Arksey and O'Malley's scoping review steps, which include identifying the research question, identifying relevant studies, study selection, charting the data and collating, summarising and reporting. ${ }^{31}$ A scoping review has been selected as the most appropriate review type as it enables a means to synthesise the breadth and type of literature available and provides a framework for both mapping the literature, recording the types of studies that have been conducted and identifying any gaps that may exist in the literature. ${ }^{31}$

\section{Step 1: identifying the research question}

This scoping review aims to explore, synthesise and evaluate the literature on rural LIC programmes and rural medical workforce outcomes. The population, exposure and outcomes tool has been used to help formulate the research question; ${ }^{32}$

Population: medical doctors

Exposure: rural LIC

Outcomes: medical workforce geographic locations (rural/metropolitan) and medical specialty.

The research question is what literature is available on the geographic workforce outcomes of medical graduates who have participated in a rural LIC programme. Specifically:

1. What literature is available on the workforce outcomes (practice location and medical specialty) of medical graduates who have participated in a rural LIC programme?

2. How have workforce outcomes (practice location and medical specialty) of rural LIC programmes been described in the literature?

3. What elements of rural LIC medical programme design have been shown to be positively associated with graduates working rurally?

4. What gaps exist in the current literature?

\section{Step 2: identify relevant studies}

The search strategy will aim to locate published peerreviewed literature. A preliminary search of MEDLINE and CINAHL was conducted to identify literature on the topic. Known articles on the topic were used to extract appropriate search terms contained in both the titles and abstracts and the index terms used to describe the articles were then applied to develop a full search (online supplemental file 1). The search strategy, including all identified keywords and index terms will be adapted for each individual database which will include MEDLINE, CINAHL complete (EBSCOhost), Scopus, Embase (Elsevier) and ISI Web of Science. The reference lists and journal sites of articles selected for full-text reviews will also be screened and searched to aid in the identification of additional papers.

\section{Inclusion criteria}

The population in the studies will be medical doctors who have participated in a rural LIC programme, either during an undergraduate or graduate medical degree. Participants will be registered medical practitioners, with a recognised geographic work location that has been classified as either rural or metropolitan (comparator group) by the study's authors. There will be no date range imposed on literature as foundation rural LIC programmes such as the Minnesota Rural Physician Associate Programme have been established since the 1970's.

The exposure will be participation in a rural LIC programme. For inclusion, studies will need to meet the typology of a LIC as defined by the CLIC. ${ }^{12}$ Papers that either self-identify as a LIC programme, meet the CLIC criteria or are a recognised LIC programme name associated with the CLIC network will be included. ${ }^{11}$ Although programmes may vary, fundamental components recommended by the CLIC network to be recognised as a LIC must be present, including (i) medical students participating in the comprehensive care of patients' overtime, (ii) medical students having continuing relationships with these patients clinicians and (iii) medical students meeting most the year's core clinical competencies across multiple discplines simultaneously. ${ }^{12}$ To adhere to the third criteria, the duration of the programme must be for 6 months or more.

The LIC setting must be a rural location. Rural locations will be identified and defined by the authors using a rural classification system of the country or jurisdiction of the LIC programme. For example, in Australia, geographic classifications are reported by the Australian Statistical Geography Standard Remoteness Area and/ or the Modified Monash Model, which employ 5-point and 7-point classification systems, respectively, on level of remoteness and population size. ${ }^{33}$ Definitions and/ or classifications systems used in studies will be extracted to describe both the rural LIC locations and the medical workforce outcomes. There are inconsistencies in how health research reports geographic locations; therefore, these reviews will align with the emerging recommendation for reviews to present all available geographic categories and then combine as required to answer the research question. ${ }^{35}$

The outcomes of the included studies will be the geographic and medical specialities of graduates from rural LIC programmes. Medical workforce outcomes are defined as the graduates' geographic work location 
and information pertaining to the vocational medical specialty they have either enrolled in, are training in or have completed. Studies will include medical workforce outcomes of rural LIC graduates at any stage of their medical career; prevocational, vocational, or post fellowship.

If it is unclear whether the programme is a LIC or is in a rural location; the corresponding authors will be contacted for further information. If clarification is not forthcoming, the study will be excluded from the review.

\section{Exclusion criteria}

Studies from LIC programmes based in metropolitan settings will not be included unless they are compared with rural LIC programmes and results are reported separately.

A 2016 study by Worley et al identified three distinct clusters of LIC programmes, namely comprehensive LICs, blended LICs and LIC-like amalgamative clerkships. ${ }^{10}$ Programmes considered to be LIC-like amalgamative clerkships, do not meet the third CLIC criteria as students only spent between 6 and 18 weeks (less than $50 \%$ of academic year) in these clerkships. As longitudinal duration is a core component required to meet the LIC inclusion, LIC-like studies will be excluded if the duration of the programme is less than 6 months.

Studies reporting on the rural workforce intentions of LIC medical students will not be included due to the complexities associated with reporting on the validity of such rural intentions translating to actual workforce outcomes. ${ }^{36}$ Furthermore, studies reporting on postgraduate training in LIC like programmes will not be included as postgraduate training is very different to that undertaken in medical school. Participants will not be excluded from the study based on any demographic data such as age, gender, marital status, ethnicity or country in which the LIC programme is undertaken.

Due to resource limitations articles written in languages other than English will be excluded.

\section{Step 3: selection of studies}

After the search of all databases, the identified records will be collated and uploaded into Endnote X9 (Clarivate Analytics, PA, USA) with duplicates removed. Two independent reviewers will then screen and assess the titles and abstracts of all papers against the predetermined inclusion criteria using Covidence software. ${ }^{37}$ Papers deemed to meet these criteria will be retrieved in full. In the event the full-text version of the article is unavailable, the corresponding authors will be contacted to request the fulltext version. If the full-text version is not forthcoming, the article will be excluded. Citations of the accepted fulltext versions will be reviewed against the inclusion criteria by both reviewers, independently. Reasons why full-text papers are excluded will be recorded and reported in the review. Any disagreements that arise between the reviewers during the selection process will be resolved through discussion, or by a third reviewer. The results of the search will be comprehensively detailed in the final review and presented in a PRISMA-ScR flow diagram.

This review will consider all types of quantitative and mixed-methods study designs for inclusion.

\section{Step 4: data extraction and charting}

Data will be extracted from papers included in the scoping review by two independent reviewers. A data extraction tool will be used to facilitate this process and ensure appropriate and standardised information is being extracted by both reviewers (online supplemental file 2).

To synthesise and allow for meaningful interpretation of the medical workforce outcomes data (geographic work location and/medical specialty), information where available will be extracted on the length of LIC programme, type of medical degree (undergraduate or graduate entry), length of medical degree, year of LIC programme within the medical degree, country/location of LIC, country's definition of rurality, setting of LIC, LIC programme name, LIC selection or student preference policies and postgraduate year of LIC graduates. Any information on other forms of medical school training LIC graduates have undertaken will also be extracted, for example, locations and types of clerkships undertaken pre-LIC or post-LIC year.

Any other variables that are used in the analysis of rural LIC graduate workforce outcomes such as rural background, gender, age, rural scholarship, type of medical place (eg, bonded, international and domestic) and marital status will also be extracted and presented.

The methodology and statistical analysis employed within the studies will be extracted and charted thematically to allow for meaningful interpretation of the data.

Furthermore, author names, associated institutions and years of publication will be recorded. A draft extraction tool is provided (online supplemental file 2). The draft extraction tool will be modified and revised as required during the process of extracting data from each included paper. Any modifications made will be detailed in the full scoping review. Any disagreements that arise between the reviewers in relation to data extraction will be resolved through discussion, or with a third reviewer. Authors of papers will be contacted to request missing or additional data, where required.

\section{Step 5: collating, summarising, and reporting}

The study selection process will be illustrated in a flow chart aligned with PRISMA-Scr. The quantitative results generated by the extraction tool will be presented in diagrammatic or tabular form. A preliminary search of the literature has identified predominately cross-sectional, observational, and retrospective quantitative studies.

As the quantitative studies are heterogeneous, a narrative synthesis will be used to discuss literature on rural LIC programmes and rural workforce outcomes, identifying gaps in the literature and making recommendations on areas that require further research. 


\section{PATIENT AND PUBLIC INVOLVEMENT}

No patients or the public were involved in the preparation of this scoping review protocol. Participants in the study are medical doctors who have completed a rural LIC. Results from this scoping review will be used to inform a qualitative study that will aim to provide a deeper understanding of their experience within this clerkship model.

\section{ETHICS AND DISSEMINATION}

The study does not involve primary data collection, therefore does not require formal ethics approval from an ethics board.

Results from this scoping review will be submitted to either a peer-reviewed medical education or rural health journal. Presentations of the findings may also be undertaken at medical education or rural health conferences. The review will also be shared within the LIC community of medical educators.

\section{DISCUSSION}

This scoping review will explore workforce outcomes of rural LIC programmes, charting how this has been previously researched. The review will follow the scoping review frameworks established by PRISMA-Scr guidelines and the six steps by Arksey and O'Malley. ${ }^{30}{ }^{31}$ Any amendments that are required during the review process will be clearly recorded.

Limitations within this scoping review include the absence of resources to include articles written in languages other than English. Variations in the terminology and definitions of rurality between countries, states and jurisdictions may limit the inclusion of some studies and limit the ability for direct comparisons to be drawn. To mitigate this, all studies definitions of rurality will be extracted and recorded. Similarly, some LIC clerkships could potentially be omitted if the key elements required to be considered a LIC are not transparently described.

As LIC programmes vary, those that meet the inclusion criteria will be compared to determine similarities and differences in clerkship structures and authors' conclusions on programme design that may influence rural workforce outcomes. Synthesis of this literature should enable clear mapping on how workforce outcomes of rural LICs have been studied and what gaps exist in the literature.

\section{Twitter Jessica Beattie @JessicaBeatti15 and Lara Fuller @larafuller00}

Acknowledgements We acknowledge the contribution of Deakin University librarian Julie Higgins in providing guidance on the development of the initial search strategy and use of academic databases and information sources. Authors are funded by the Rural Health Multidisciplinary Training (RHMT) Program (Australian Department of Health).

Contributors All authors contributed to the design of this protocol. JB initiated the project. The protocol was drafted by JB and was refined by MJB and LF. Protocol advice was provided by MB. All authors contributed to the manuscript and read and approved the final copy.
Funding The authors have not declared a specific grant for this research from any funding agency in the public, commercial or not-for-profit sectors.

Competing interests None declared.

Patient consent for publication Not required.

Provenance and peer review Not commissioned; externally peer reviewed.

Supplemental material This content has been supplied by the author(s). It has not been vetted by BMJ Publishing Group Limited (BMJ) and may not have been peer-reviewed. Any opinions or recommendations discussed are solely those of the author(s) and are not endorsed by BMJ. BMJ disclaims all liability and responsibility arising from any reliance placed on the content. Where the content includes any translated material, BMJ does not warrant the accuracy and reliability of the translations (including but not limited to local regulations, clinical guidelines, terminology, drug names and drug dosages), and is not responsible for any error and/or omissions arising from translation and adaptation or otherwise.

Open access This is an open access article distributed in accordance with the Creative Commons Attribution Non Commercial (CC BY-NC 4.0) license, which permits others to distribute, remix, adapt, build upon this work non-commercially, and license their derivative works on different terms, provided the original work is properly cited, appropriate credit is given, any changes made indicated, and the use is non-commercial. See: http://creativecommons.org/licenses/by-nc/4.0/.

ORCID iD

Jessica Beattie http://orcid.org/0000-0003-3339-1418

\section{REFERENCES}

1 Australian Parliament. The factors affecting the supply of health services and medical professionals in rural areas. Canberra: Australian Government, 2012.

2 Dussault G, Franceschini MC. Not enough there, too many here: understanding geographical imbalances in the distribution of the health workforce. Hum Resour Health 2006;4:12.

3 Canadian Medical Association, Physician Data Centre, Physician Data Centre. CMA, 2022.

4 Australian Government, Department of Health. National medical workforce strategy. scoping framework. Canberra, 2019.

5 U.S Department of Health and Human Services. State variability in supply of office-based primary care providers: United States, 2012 NCHS data brief, 2014.

6 Basu S, Berkowitz SA, Phillips RL, et al. Association of primary care physician supply with population mortality in the United States, 2005-2015. JAMA Intern Med 2019;179:506-14.

7 Australian Institute of Health and Welfare. Australia's Health 2018. Canberra, 2018.

8 Australian Government. Rural health multidisciplinary training (RHMT) program framework 2019-2020. Canberra: Department of Health, 2019.

9 Ellaway R, Graves L, Berry S, et al. Twelve tips for designing and running longitudinal integrated clerkships. Med Teach 2013;35:989-95.

10 Worley P, Couper I, Strasser R, et al. A typology of longitudinal integrated clerkships. Med Educ 2016;50:922-32.

11 Hudson JN, Knight PJ, Weston KM. Patient perceptions of innovative longitudinal integrated clerkships based in regional, rural and remote primary care: a qualitative study. BMC Fam Pract 2012;13:72.

12 Consortium of Longitudinal Integrated Clerkships. Core elements, 2020. https://clicmeded.com/

13 Walters L, Greenhill J, Richards J, et al. Outcomes of longitudinal integrated clinical placements for students, clinicians and society. Med Educ 2012;46:1028-41.

14 Thistlethwaite JE, Bartle E, Chong AAL, et al. A review of longitudinal community and hospital placements in medical education: BEME guide No. 26. Med Teach 2013;35:e1340-64.

15 Stupart D, Beattie J, Lawson M, et al. Medical students can learn surgery effectively in a rural longitudinal integrated clerkship. J Surg Educ 2020;77:1407-13.

16 Fuller L, Lawson M, Beattie J. The impact of clerkship model and clinical setting on medical student's participation in the clinical workplace: a comparison of rural LIC and rural block rotation experience. Med Teach 2021;43:307-13.

17 Caygill R, Peardon M, Waite C, et al. Comparing a longitudinal integrated clerkship with traditional hospital-based rotations in a rural setting. Med Teach 2017;39:520-6.

18 O'Brien BC, Poncelet AN, Hansen L, et al. Students' workplace learning in two clerkship models: a multi-site observational study. Med Educ 2012;46:613-24. 
19 Hauer KE, Hirsh D, Ma I, et al. The role of role: learning in longitudinal integrated and traditional block clerkships. Med Educ 2012;46:698-710.

20 Norris TE, Schaad DC, DeWitt D, et al. Longitudinal integrated clerkships for medical students: an innovation adopted by medical schools in Australia, Canada, South Africa, and the United States. Acad Med 2009;84:902-7.

21 O'Sullivan BG, McGrail MR, Russell D, et al. A review of characteristics and outcomes of Australia's undergraduate medical education rural immersion programs. Hum Resour Health 2018;16:8

22 Kitchener S, Day R, Faux D, et al. Longlook: initial outcomes of a longitudinal integrated rural clinical placement program. Aust J Rural Health 2015;23:169-75.

23 O'Sullivan B, McGrail M, Russell D, et al. Duration and setting of rura immersion during the medical degree relates to rural work outcomes. Med Educ 2018;52:803-15.

24 Myhre DL, Bajaj S, Woloschuk W. Practice locations of longitudinal integrated clerkship graduates: a matched-cohort study. Can J Rural Med 2016;21:13-16.

25 Campbell DG, McGrail MR, O'Sullivan B, et al. Outcomes of a 1-year longitudinal integrated medical clerkship in small rural Victorian communities. Rural Remote Health 2019;19:4987.

26 Halaas GW, Zink T, Finstad D, et al. Recruitment and retention of rural physicians: outcomes from the rural physician associate program of Minnesota. J Rural Health 2008;24:345-52.

27 Stagg P, Prideaux D, Greenhill J, et al. Are medical students influenced by preceptors in making career choices, and if so how? A systematic review. Rural Remote Health 2012:12.
28 Bartlett M, Couper I, Poncelet A, et al. The do's, don'ts and don't knows of establishing a sustainable longitudinal integrated clerkship. Perspect Med Educ 2020;9:5-19.

29 Brown ME, Anderson K, Finn GM. A narrative literature review considering the development and implementation of longitudinal integrated clerkships, including a practical guide for application. $J$ Med Educ Curric Dev 2019;6:238212051984940.

30 Tricco AC, Lillie E, Zarin W, et al. PRISMA extension for scoping reviews (PRISMA-ScR): checklist and explanation. Ann Intern Med 2018;169:467-73.

31 Arksey H, O'Malley L. Scoping studies: towards a methodological framework. Int J Soc Res Methodol 2005;8:19-32.

32 Aromataris E, Munn Z. JBI manual for evidence synthesis. JBI, 2020.

33 Australian Bureau of Statistics. The Australian statistical geographical classification (ASGC) remoteness structure. Canberra, 2016.

34 Modified Monash Model. Australian department of health (2019). Available: https://www.health.gov.au/sites/ default/files/ documents/2020/07/modified-monas h-model -fact- sheet.pdf

35 Versace VL, Beks H, Charles J. Towards consistent geographic reporting of Australian health research. Med J Aust 2021;215:525.

36 Sen Gupta T, Murray R, Hays R, et al. James Cook University MBBS graduate intentions and intern destinations: a comparative study with other Queensland and Australian medical schools. Rural Remote Health 2013.

37 Veritas Health Innovation. Covidence systematic review software. Melbourne, Australia, 2021 